\title{
Laser annealed in-situ P-doped Ge for on-chip laser source applications (Conference Presentation)
}

\author{
Ashwyn Srinivasan, Marianna Pantouvaki, Yosuke Shimura, Clement Porret, IMEC \\ (Belgium); Rik Van Deun, Univ. Gent (Belgium); Roger Loo, IMEC (Belgium); Dries Van \\ Thourhout, Univ. Gent (Belgium); Joris Van Campenhout, IMEC (Belgium)
}

\begin{abstract}
Realization of a monolithically integrated on-chip laser source remains the holy-grail of Silicon Photonics. Germanium $(\mathrm{Ge})$ is a promising semiconductor for lasing applications when highly doped with Phosphorous (P) and or alloyed with Sn [1,2]. P doping makes Ge a pseudo-direct band gap material and the emitted wavelengths are compatible with fiber-optic communication applications. However, in-situ P doping with Ge2H6 precursor allows a maximum active $\mathrm{P}$ concentration of $6 \times 1019 \mathrm{~cm}-3$ [3]. Even with such active $\mathrm{P}$ levels, $\mathrm{n}++\mathrm{Ge}$ is still an indirect band gap material and could result in very high threshold current densities. In this work, we demonstrate P-doped Ge layers with active n-type doping beyond $1020 \mathrm{~cm}-3$, grown using Ge2H6 and PH3 and subsequently laser annealed, targeting power-efficient on-chip laser sources.

The use of Ge2H6 precursors during the growth of P-doped Ge increases the active P concentration level to a record fully activated concentration of $1.3 \times 1020 \mathrm{~cm}-3$ when laser annealed with a fluence of $1.2 \mathrm{~J} / \mathrm{cm} 2$. The material stack consisted of $200 \mathrm{~nm}$ thick P-doped Ge grown on an annealed $1 \mu \mathrm{m}$ Ge buffer on Si. Ge:P epitaxy was performed with $\mathrm{PH} 3$ and $\mathrm{Ge} 2 \mathrm{H} 6$ at $320 \mathrm{oC}$. Low temperature growth enable Ge:P epitaxy far from thermodynamic equilibrium, resulting in an enhanced incorporation of $\mathrm{P}$ atoms [3]. At such high active $\mathrm{P}$ concentration, the $\mathrm{n}++$ Ge layer is expected to be a pseudo-direct band gap material. The photoluminescence (PL) intensities for layers with highest active P concentration show an enhancement of $18 \times$ when compared to undoped Ge grown on Si as shown in Fig. 1 and Fig. 2. The layers were optically pumped with a $640 \mathrm{~nm}$ laser and an incident intensity of $410 \mathrm{~mW} / \mathrm{cm} 2$. The PL was measured with a NIR spectrometer with a Hamamatsu R5509-72 NIR photomultiplier tube detector whose detectivity drops at $1620 \mathrm{~nm}$. Due to high active $\mathrm{P}$ concentration, we expect band gap narrowing phenomena to push the PL peak to wavelengths beyond the detection limit $(1620 \mathrm{~nm})$ of the setup. Therefore, the $18 \times$ enhancement is a lower limit estimation. In this contribution, an extensive study of laser annealing conditions and their impact on material properties will be discussed.

A major concern in using highly doped Ge as an active medium is the increase in free-carrier absorption (FCA). However, results reported in [4] suggest that FCA is significantly dominated by holes due to larger absorption crosssection of holes compared to electrons. The FCA results in [4] and JDOS modeling were used to calculate the gain spectrum for the highest doped Ge samples, including the typical $0.25 \%$ biaxial tensile strain of epitaxial Ge on Si. A carrier lifetime of $3 \mathrm{~ns}$ is required as shown in Fig. 3 for a target threshold current density of sub- $20 \mathrm{kA} / \mathrm{cm} 2$ which represents at least tenfold reduction when compared to active P-doping level of $6 \times 1019 \mathrm{~cm}-3$. As a result, laser annealed highly doped Ge layers grown with $\mathrm{Ge} 2 \mathrm{H} 6$ precursors are a promising approach for realizing a power efficient on-chip Ge laser source.
\end{abstract}

